\title{
Calculation of beams with corrugated wall on the stability of a flat bending shape
}

\author{
Anastasia Lapina, Ivan Zotov, Anton Chepurnenko * and Batyr Yazyev \\ Don State Technical University, 162 Sotcialisticheskaya str., 344022, Russia
}

\begin{abstract}
The article presents the method of calculating beams with a corrugated wall on the stability of a flat bending shape. The problem reduces to a differential equation of fourth order with respect to the twist angle. The solution is performed numerically by the finite difference method. A comparison of the results with the software package LIRA is presented.
\end{abstract}

\section{Introduction}

I-beams with corrugated walls are actively used in modern construction. In the literature there are a large number of works on such structures, including [1-4]. Most of them are devoted to the study of the stress-strain state. Finite element complexes make it possible to model beams with a corrugated wall with regard to their real geometry, but this approach is rather laborious. Therefore, there is a need for an approximate method suitable for engineering calculations.

\section{Methods}

We will use the general V.Z. Vlasov theory of thin-walled rods of an open profile [5] to solve the problem of lateral buckling of a beam with a corrugated wall. The relationship between the torque $M_{\mathrm{t}}$ and the twisting angle $\theta$ for a thin-walled rod has the form [6]:

$$
-E I_{\omega} \frac{d^{3} \theta}{d x^{3}}+G I_{t} \frac{d \theta}{d x}=M_{t},
$$

where $I_{\omega}$ is the sectorial moment of inertia, $I_{t}$ is the moment of inertia during torsion, $E$ and $G$ are the elastic moduli of the first and second kind, respectively.

The cross section of the corrugated beam is shown in fig. 1.

\footnotetext{
* Corresponding author: anton_chepurnenk@ mail.ru
} 


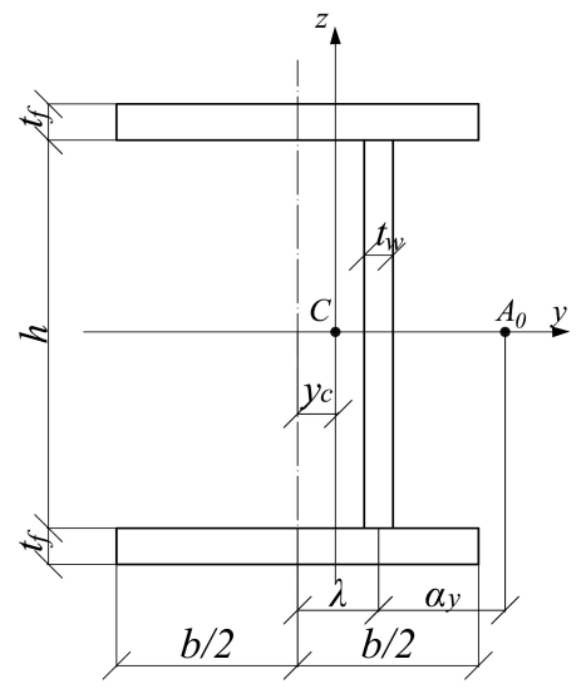

Fig. 1. Cross section of corrugated wall beam

For a profile consisting of $n$ rectangles, the value $I_{\mathrm{t}}$ is calculated by the formula [7]:

$$
I_{t}=\frac{1}{3} \gamma \sum_{i=1}^{n} s_{i} t_{i}^{3}
$$

where $s_{\mathrm{i}}$ and $t_{\mathrm{i}}$ are respectively the length and thickness of the $i$-th wall; $\gamma$ is a coefficient that depends on the shape of the cross section.

Applied to a corrugated beam, the formula (2) takes the form:

$$
I_{t}=\frac{1}{3}\left(h t_{w}^{3}+2 b t_{f}^{3}\right) \text {. }
$$

The position of the center of gravity of the cross section can be calculated by the formula:

$$
y_{c}=\frac{\lambda t_{w} h}{2 t_{f} b+t_{w} h} .
$$

The moment of inertia of the section relative to the main central axis $z$ is calculated by the formula:

$$
I_{z}=2\left[\frac{t_{f} b^{3}}{12}+t_{f} b y_{c}^{2}\right]+\frac{h t_{w}^{3}}{12}+t_{w} h\left(\lambda-\frac{\lambda t_{w} h}{2 t_{f} b+t_{w} h}\right)^{2}
$$

The center of the bending of the section $A_{0}$ is located at a distance $\alpha_{\mathrm{y}}$ from the wall, the value of which is calculated by the formula:

$$
\alpha_{y}=\frac{t_{f} b \lambda h^{2}}{2 I_{y}}
$$

where $I_{y}=\frac{t_{w} h^{3}}{12}+\frac{2 b t_{f}^{3}}{12}+\frac{b t_{f} h^{2}}{2}-$ axial moment of inertia of the section. 
The main sectorial moment of inertia of a section is determined as follows:

$$
I_{\omega}=\frac{t_{f} b h^{2}}{6}\left[\left(\frac{b}{2}+\lambda-\alpha_{y}\right)^{2}+\left(\frac{b}{2}-\lambda+\alpha_{y}\right)^{2}-\left(\frac{b}{2}+\lambda-\alpha_{y}\right)\left(\frac{b}{2}-\lambda+\alpha_{y}\right)\right]+\frac{t_{w} h^{3} \alpha_{y}^{2}}{12} .
$$

The geometric characteristics of $I_{\mathrm{z}}$ and $I_{\omega}$ depend on $\lambda$, which in turn is a function of $x$. The value of $I_{\mathrm{t}}$ is not dependent on the $x$ coordinate.

To determine the critical load, we use the static Euler criterion. The beam element at the moment of buckling is shown in fig. 2 (the wall is conventionally shown smooth). At the moment of buckling, a torque $M_{x}$ occurs in the beam. Writing the sum of moments relative to the $x$ axis of the beam in the undeformed state, we get:

$$
M_{x}+d M_{x}+q d x\left(\frac{v+(v+d v)}{2}+a \theta\right)-M_{x}=0
$$

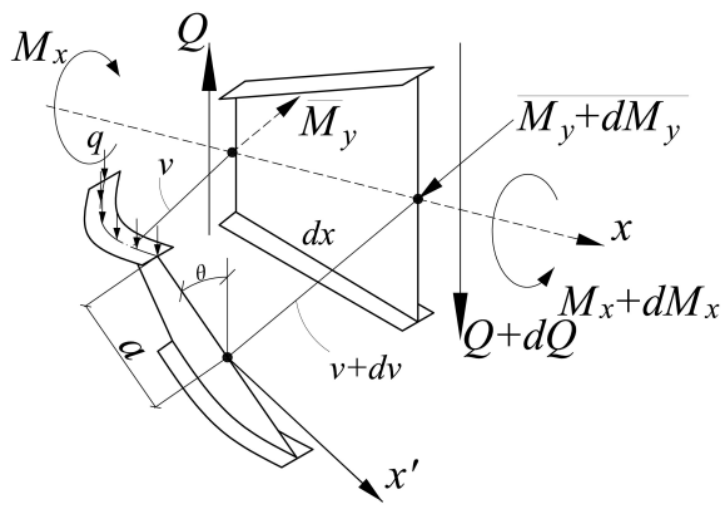

Fig. 2. Beam element at the moment of buckling

Neglecting members of a higher order of smallness, the expression (8) can be represented as:

$$
\frac{d M_{x}}{d x}=-q(v+a \theta)
$$

Torque on the $x^{\prime}$ axis is calculated as:

$$
M_{x^{\prime}}=M_{t}=M_{x}-Q v+M_{y} \frac{d v}{d x} .
$$

Differentiating expression (10) by $x$, we get:

$$
\frac{d M_{t}}{d x}=\frac{d M_{x}}{d x}-\frac{d Q}{d x} v-Q \frac{d v}{d x}+\frac{d M_{y}}{d x} \frac{d v}{d x}+M_{y} \frac{d^{2} v}{d x^{2}} .
$$

Substituting (9) into (11) and given that $\frac{d Q}{d x}=-q$ and $\frac{d M_{y}}{d x}=Q$, we get:

$$
\frac{d M_{t}}{d x}=M_{y} \frac{d^{2} v}{d x^{2}}-q a \theta .
$$

The bending moment relative to the axis $z^{\prime}$ at the moment of buckling is defined as: 


$$
M_{z^{\prime}}=M_{y} \theta=-E I_{z} \frac{d^{2} v}{d x^{2}}
$$

We differentiate equality (1) with respect to $x$ :

$$
\frac{d M_{t}}{d x}=-E I_{\omega} \frac{d^{4} \theta}{d x^{4}}-E \frac{d I_{\omega}}{d x} \frac{d^{3} \theta}{d x^{3}}+G I_{t} \frac{d^{2} \theta}{d x^{2}} .
$$

Expressing from (13) the second derivative of the deflection and substituting further the value $\frac{d^{2} v}{d x^{2}}$ in (12) and then equating the right-hand sides (14) and (12), we obtain the basic resolving equation:

$$
-E I_{\omega} \frac{d^{4} \theta}{d x^{4}}-E \frac{d I_{\omega}}{d x} \frac{d^{3} \theta}{d x^{3}}+G I_{t} \frac{d^{2} \theta}{d x^{2}}+\theta\left(\frac{M_{y}^{2}}{E I_{z}}+q a\right)=0
$$

Thus, the problem has been reduced to a fourth-order differential equation with respect to the twist angle.

We will consider the calculation method on the example of a hinged at the ends beam under a uniformly distributed load (fig. 3). In the calculation, we assume that the rotation of the beam about its $x$ axis is excluded in the reference sections, and also that in the endsection deplanation occurs freely, i.e. they do not have a bimoment $B$. The corresponding boundary conditions at $x=0$ and $x=l$ are written in the form:

$$
\theta=0 ; \quad B=-E I_{\omega} \frac{d^{2} \theta}{d x^{2}}=0 \rightarrow \frac{d^{2} \theta}{d x^{2}}=0 .
$$

The solution of equation (15) is performed by the finite difference method. Finally, the problem is reduced to a homogeneous system of linear algebraic equations:

$$
([A]-t[B]-\sqrt{t} a[E])\{X\}=0,
$$

where $[E]$ is a unit matrix, $t=q^{2},\{X\}=\left\{\begin{array}{llll}\theta_{2} & \theta_{3} & \ldots & \theta_{n}\end{array}\right\}^{T}$. Matrices $[A]$ and $[B]$ are not given here because of their bulkiness.

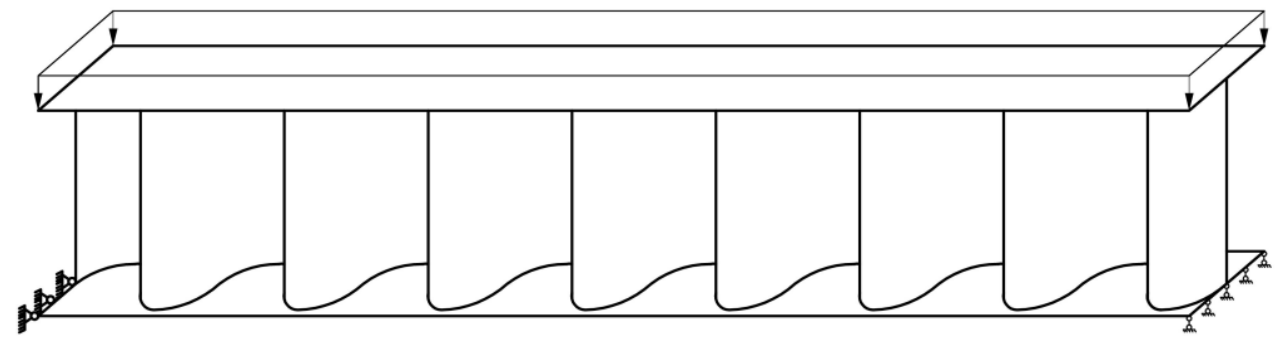

Fig. 3. Calculation scheme

The critical load is determined from the condition that the determinant of the system (17) is zero: 


$$
|[A]-t[B]-\sqrt{t} a[E]|=0 .
$$

The method of solving equations of the form (18) is given in $[8,9]$.

\section{Results and Discussion}

The test problem for a beam with sinusoidal corrugation $\lambda=f \sin (2 \pi x / \varepsilon)$ was solved at $b=0.1 \mathrm{~m}, h=0.3 \mathrm{~m}, t_{w}=3 \mathrm{~mm}, t_{f}=4 \mathrm{~mm}, \varepsilon=5 \mathrm{~cm}, f=3 \mathrm{~cm}$. The span of the beam was taken to be $l=3 \mathrm{~m}$. Calculations were performed in a package Matlab. The critical load according to the results of the calculation was $19.2 \mathrm{kN} / \mathrm{m}$.

The same problem was solved in a three-dimensional formulation in the Lira-SAPR 2013 software package. To eliminate local stability loss in the support areas, support ribs were installed. The wall and supports ribs were modeled with flat rectangular finite elements of shell, and the shelves were modeled with flat triangular finite elements. The form of buckling is shown in Fig.4. The critical load according to the results of the calculation in the program complex was $20.1 \mathrm{kN} / \mathrm{m}$. The solution based on the theory of thin-walled rods differs from this value by $4.5 \%$.

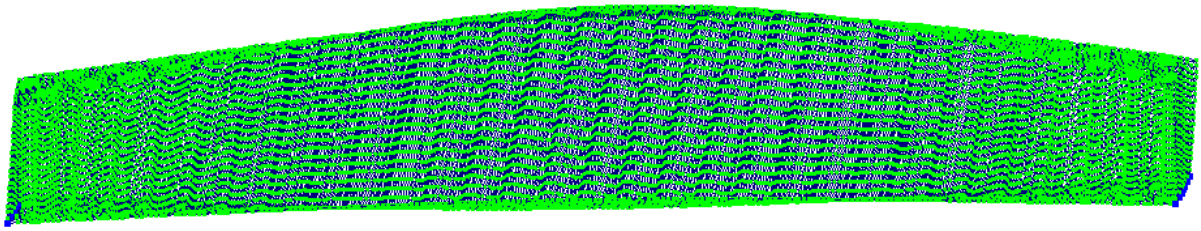

$\mathrm{Z}_{\mathrm{Z} X}$

Fig. 4. Form of buckling for a beam with corrugated wall

Also for comparison, a calculation was made on the stability of a beam with smooth wall. When calculated in the Lira-SAPR software package, the critical load was $15.39 \mathrm{kN} / \mathrm{m}$, and 15.74 $\mathrm{kN} / \mathrm{m}$ when calculated in the Matlab package. Form of buckling for a beam with smooth wall is shown in Fig. 5.

Buckling mode in global system 1

Stab. factor 15.3864

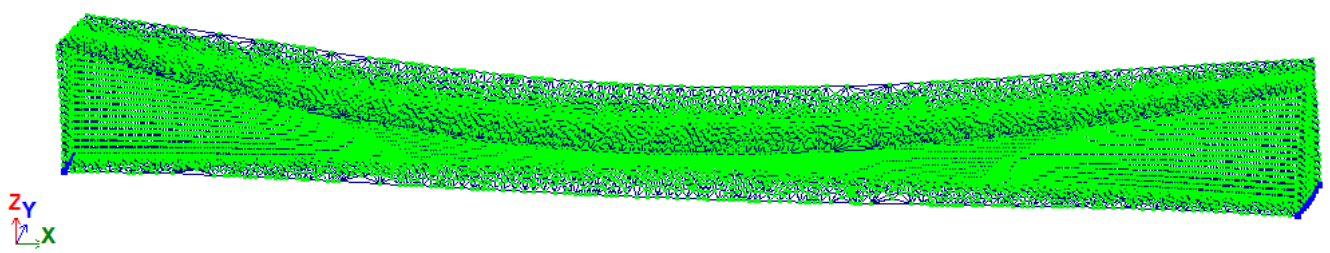

Fig. 5. Form of buckling for a beam with smooth wall 


\section{Summary}

A method for calculating corrugated wall beams based on the theory of thin-walled rods of V.Z. Vlasov was developed. For test problems, a good agreement of results was obtained with a solution in a three-dimensional formulation using FEM. Compared with a beam with a smooth wall for a structure with a corrugated wall, the critical load was higher by $30.6 \%$

\section{References}

1. A. O. Lukin, V. Y. Alpatov, Materials Science Forum, 931 (2018) 247-251

2. A. A. Lukin, I. S. Kholopov, Procedia Engineering, 153 (2016) 414-418.

3. A.O. Lukin, A.A. Suvorov, Construction of Unique Buildings and Structures, 2 (2016) 45-67.

4. I. M. Balzannikov, I.S. Kholopov et al., Procedia Engineering. 111 (2015) 74-81.

5. V.Z. Vlasov, Thin-Walled Elastic Rods (Fizmatgiz, 1959)

6. A. S. Volmir, Stability of deformable systems (Nauka, 1967)

7. S. P. Timoshenko, J. M. Gere, Theory of elastic stability (McGraw-Hill, 1961)

8. A.S. Chepurnenko, V.V. Ulianskaya, D.A. Vysokovsky, I.M. Zotov, Inženernyj vestnik Dona, 2 (2018)

9. A.S. Chepurnenko, V.V. Ulianskaya, S.B. Yazyev, I.M. Zotov, MATEC, 196 (2018) 01003 